\title{
Lecture Capture using Large Interactive Display Systems
}

\author{
Mark Apperley, Sam Jansen, Amos Jeffries, Masood Masoodian, Laurie McLeod, \\ Lance Paine, Bill Rogers, Kirsten Thomson, Tony Voyle \\ Department of Computer Science \\ University of Waikato \\ Hamilton, New Zealand \\ M.Masoodian@cs.waikato.ac.nz
}

\begin{abstract}
There are various software technologies that allow capture and redelivery of lectures. Most of these technologies however rely on the use of proprietary software, often requiring extra efforts from the lecturer in terms of the initial preparation of the lecture material, or in editing and annotating after the lecture to make the material suitable for the students. To review the material students then require access to the proprietary software. This paper describes a system for the lightweight capture of lecture presentations, based on the use of a low-cost large interactive display surface, together with standard Microsoft PowerPoint ${ }^{\mathrm{TM}}$ presentation software. The captured version of the presentation includes the original lecture slides, graphical annotations made by the lecturer during the lecture, and the audio recording of the lecture; all saved as a PowerPoint file. In addition, the system adds some annotations and index slides to allow quick and easy access to different segments of the presentation. Presentations can be replayed in part or in full as required, preserving all of the content of the live lecture.
\end{abstract}

\section{1: Introduction}

Over the past few years the use of software such Microsoft PowerPoint ${ }^{\mathrm{TM}}$ has become an integral part of lecture preparation and delivery. Lecturers prepare their material using this presentation software, and then either deliver their lecture using the presentation software, or print their notes onto transparencies and use overhead projectors during the actual delivery.

One of the main reasons for the use of printed transparencies, rather than direct use of the presentation software, is because it is easier to annotate the lecture material on transparencies during the course of a lecture. Although most presentation software allows annotation of slides during presentation, it is more difficult to use a computer mouse to draw or write on a slide than it is to simply use a pen to annotate a transparency. Also, in most presentation software, such as PowerPoint ${ }^{\mathrm{TM}}$, the annotations aren't added to the slides, which means that when one moves from one slide to the next then all the annotations on the previous slide are lost. Returning to a slide means returning to the slide as it was originally stored, not as it was last seen.

A further problem in the use of existing presentation software is the lack of support for redelivery of the lecture material. Conventional presentation software at best allows one to convert the lecture notes to Web pages and put them online for students to use after the lecture. However, this is clearly less than satisfactory because the students then only get the notes without the annotations, and more importantly the audio presentation of the lecturer.

Although there is software which allows recording of lectures, both in terms of annotations and audio content, the majority of these tools place significant overheads on the lecturer to develop their material in a suitable form or using a particular application [5, 4]. These overheads often divert the presenter from the real purpose, the delivery of the content, and focus attention on the form. The effort required is typically far in excess of the preparation of the same material for live delivery.

This paper describes the LLC (Lightweight Lecture Capture) software that has been developed to support the recording of lecture presentations for review and redelivery. LLC is based on standard PowerPoint ${ }^{\mathrm{TM}}$ presentation software and requires no effort in addition to that for the original live presentation. The original presentation needs to be produced in PowerPoint ${ }^{\mathrm{TM}}$ format, and that standard application is required for the subsequent viewing of the recorded presentation. Lecture capture is best achieved through a large interactive display surface technology, while review and redelivery require only a conventional computer display and sound card.

\section{2: Large Interactive Display System}

The use of large interactive display systems in teaching and group work environments is not new. Systems such as Liveboard [3] and Smartboard [6] have been available for some time. The main problem with these technologies, however, has been their high cost that has restricted their use to mainly business meetings.

As part of an ongoing research project at the 


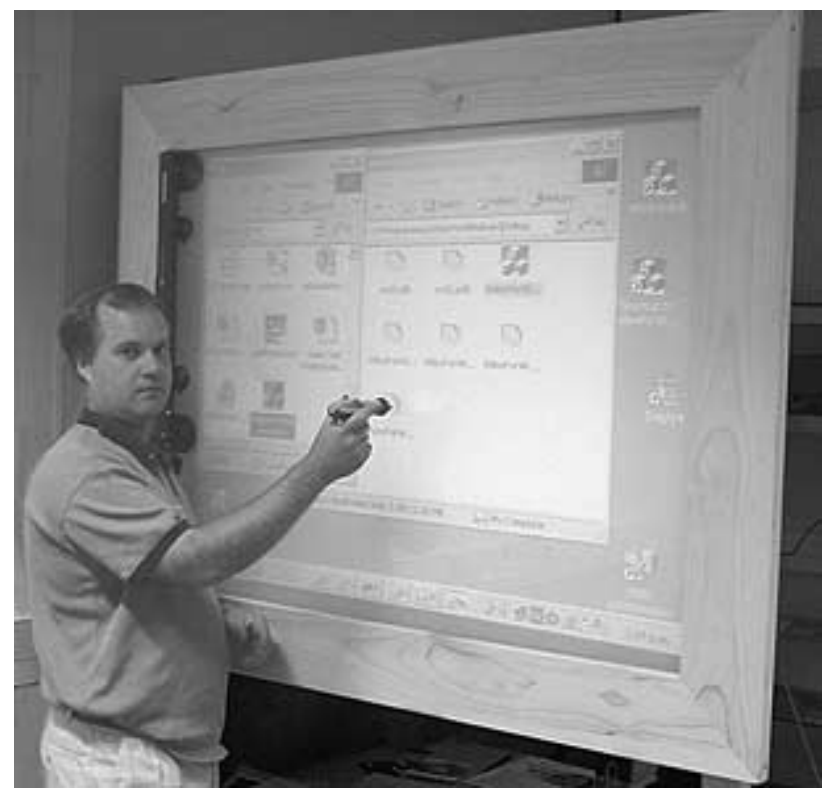

Figure 1: A prototype LIDS system in use

University of Waikato we have been working on developing a low-cost Large Interactive Display Surface (LIDS). A typical LIDS system [1] is an office whiteboard-sized screen functioning as a computer display. Attachment of $\mathrm{Mimio}^{\mathrm{TM}}$ [7] digitisation equipment at the front allows the users to directly interact with the screen using a pen, which serves a similar functionality to that of a mouse. A standard data projector and a rear projection screen are used so as to allow single user, or a small group of users, to work directly in front of the screen without occluding the image. Figure 1 shows a prototype LIDS system in use an office environment.

Even the basic LIDS system (which is just a large screen with a pen used as a mouse) is attractive to use in a number of settings such as teaching, demonstration, software evaluation and testing. The large display makes it possible for a group of people to sit and watch what is being presented to them, or alternatively they can stand around the display and take turns at contributing.

In contrast to a conventional computer and data projector setup the operation of a LIDS system is completely transparent. The person operating the system can work entirely in view of their audience. This is particularly powerful when demonstrating software or discussing usability issues. Even for the individual user the directness of object manipulation is useful. Rather than tinkering with a mouse out of view of an audience, or to the side of the display, the user actually touches object images with the pen.

Furthermore, in group work situations where the shared document presented on the screen is the focus of everyone's attention, everyone can see what is being done; creating a new interaction paradigm of "what is done is what is seen being done."

However, our objective in developing the LIDS system was not just to use it with conventional computer applications that have often been developed for single users working behind a typical computer display. Rather the aim was to trial it with specific software designed to support small group interaction in teaching or business settings. One such use of LIDS is for teaching purposes, which will be discussed here.

\section{3: Lecture Delivery Model}

Our lecture delivery model assumes that lecture notes are prepared before the presentation using standard Microsoft PowerPoint ${ }^{\mathrm{TM}}$ software. The notes can range from a simple set of outlines, perhaps just with major headings, through to a complete set of presentation slides. Alternatively, the presenter could start with a single blank slide, and simply use it as an electronic whiteboard surface; adding further slides when needed.

During the presentation the Lightweight Lecture Capture (LLC) software, which acts as an overlay to PowerPoint ${ }^{\mathrm{TM}}$, allows the presenter to make annotations and additions to the individual slides using the $\mathrm{Mimio}^{\mathrm{TM}}$ pens. These annotations are written back into the original lecture notes at each slide transition in standard PowerPoint ${ }^{\mathrm{TM}}$ format.

The presenter's voice can also be recorded by the LLC software and is written back to the original presentation file, in a similar fashion to the annotations. To get good quality recording it is best for the presenter to wear a headset microphone.

Since the annotations, additions, and audio contents are written back into the PowerPoint ${ }^{\mathrm{TM}}$ lecture notes, it is possible to further review and do basic editing of the slides after the presentation - making changes like removing accidental annotations. Some aspects of the recording are more difficult to edit with PowerPoint ${ }^{\mathrm{TM}}$ however. In particular presenters may wish to work through the audio content to improve clarity and remove irrelevant comments, coughs and hesitations. It was not the original intention to encourage such editing, because it partly negates the advantage of lightweight capture. However, user testing alerted us to the fact that some presenters would feel uncomfortable if they were unable to make corrections before publishing their recorded presentations. In response the LLC system includes a utility to allow editing of the audio recordings[2]. It is also possible that in the future other tools will be added to provide LLC with more advanced lecture-editing capabilities.

Although lecturers may use tools such as LLC for creation, recording and editing of their presentations, we see it as very important that the presentation slides are kept in a standard format so that they can be reviewed by and redelivered to students, without the students needing access to specialised software. The LLC system uses PowerPoint $^{\mathrm{TM}}$ files as its storage medium. Therefore any student with access to PowerPoint ${ }^{\mathrm{TM}}$ can review a 


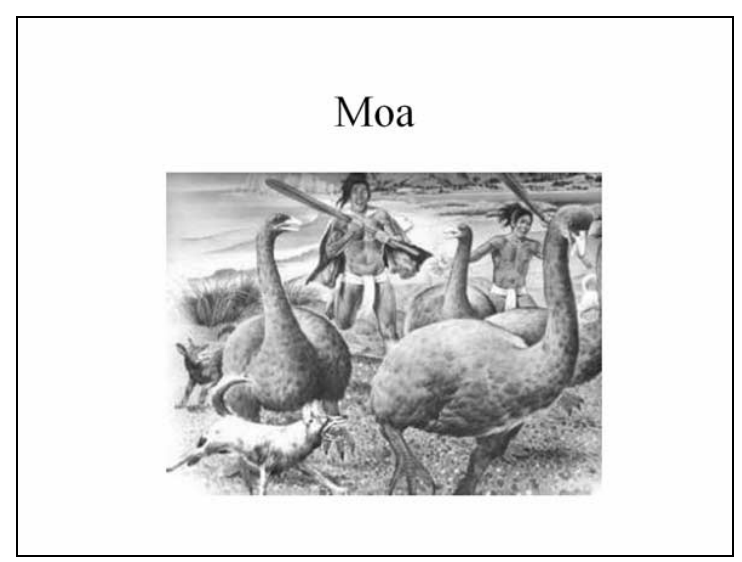

a

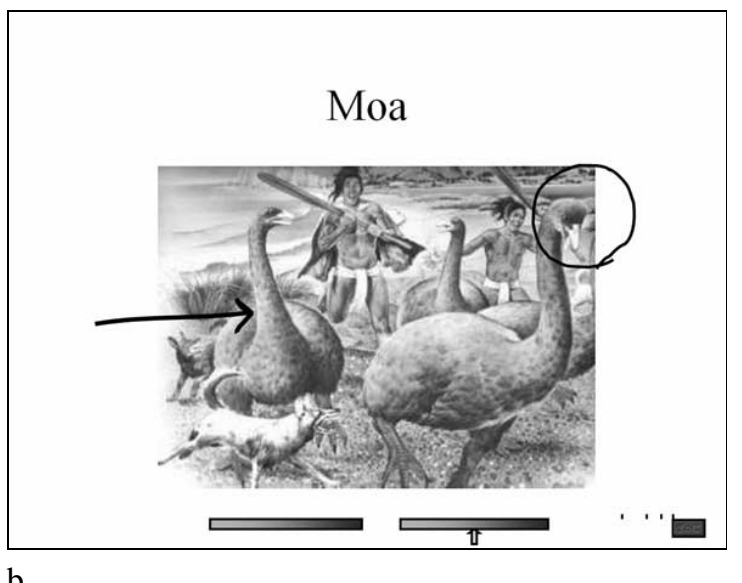

b

Figure 2: a) the original slide b) annotated slide with LLC controls added

recorded presentation. In addition the LLC system provides a utility to translate a recorded presentation into HTML, complete with audio content, although lacking animation. The HTML form can be viewed with any web browser, and can be made available over the internet from a web server if required.

\section{4: Lightweight Lecture Capture Software}

As mentioned, during lecture presentations the Lightweight Lecture Capture software operates on top of PowerPoint ${ }^{\mathrm{TM}}$, intercepting interactions and taking control of the presentation package. Once a presentation is under way, LLC intercepts Mimio $^{\mathrm{TM}}$ pen activities, recording and displaying pen strokes for later addition to the presentation. LLC also recognizes a set of pre-defined gestures; allowing the presentation to be controlled directly using the pen, rather than from a separate keyboard, mouse, or other additional device. mimic the actual pen path. Because the annotations are written into the presentation at each slide transition, when a slide is revisited during the presentation or afterwards, all of the previous annotations for that slide can be seen and replayed.

Figure 2a shows an original slide from a typical PowerPoint ${ }^{\mathrm{TM}}$ presentation, while Figure $2 \mathrm{~b}$ shows the same slide after it has been annotated by the presenter (a circle and an arrow have been added to the image). Figure $2 \mathrm{~b}$ also shows controls and indicators along the bottom of the slide that have been added by the LLC software. These controls (enlarged in Figure 3) include a timeline of the recorded audio track, showing periods of sound (bars) and periods of silence (gaps between bars). Arrows beneath the sound bars are turned on and off during replay to show which audio segment is currently playing. In Figure 3 the arrow shows that the second audio fragment is active. On replay the audio can be allowed to play automatically in sequence, or individual

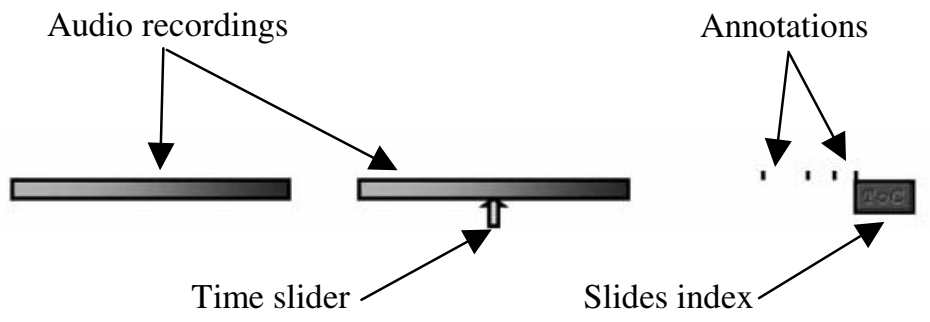

Figure 3: The controls added by LLC

When the user indicates they wish to advance to a new slide, LLC takes all of the recorded pen annotations captured for the current slide, together with the audio record, and integrates these back into the PowerPoint ${ }^{\mathrm{TM}}$ presentation for that slide. This is an important aspect of LLC, because it means that presentations can be replayed using only the standard software.

Annotations are crudely yet effectively animated in the record using PowerPoint ${ }^{\mathrm{TM}}$ entry animations, such as "wipe left" or "wipe right", chosen automatically to best audio bars can be clicked to play their part of the sound track. The small dashes above the level of the sound bars, visible in Figure 3 to the right hand side are a set of indicators showing when the slide was annotated. Each slide also includes a ToC button which links it to an additional slide inserted by LLC at the start of the presentation - the Table of Contents slide (Figure 4).

A simple gesture recognizer within LLC allows the Mimio $^{\mathrm{TM}}$ pen to be used to control the presentation. Supported gestures include a right facing arrow to 


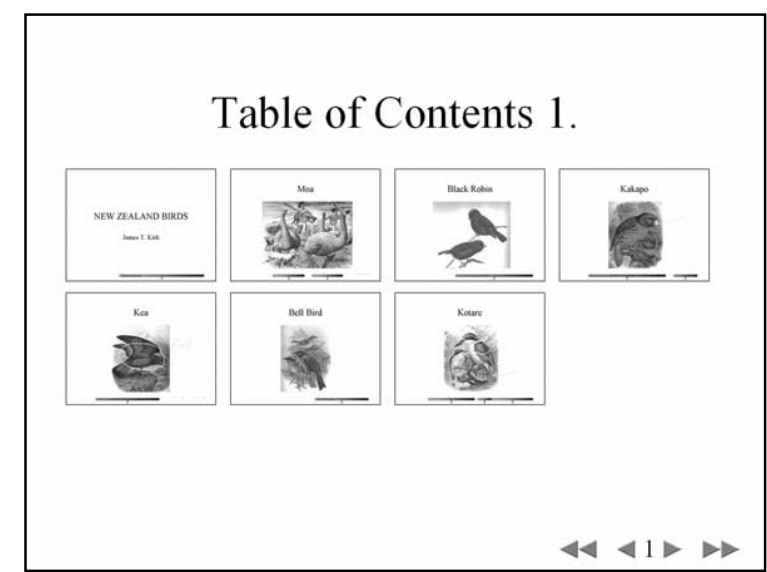

Figure 4: Table of contents generated by the LLC software

advance a slide, a left facing arrow to retreat through the presentation, and a scribble action to delete an annotation on the current slide. Gestures can also be used to edit different parts of the original PowerPoint ${ }^{\mathrm{TM}}$ slides during the presentation. A crude character recognition system, using the Grafiti character set is included on an experimental basis. This allows addition of neatly formatted text.

During review or redelivery, which can be carried out on a conventional display screen, the presentation can be indexed at the individual slide level using the standard PowerPoint $^{\mathrm{TM}}$ slide sorter view, or the LLC generated table of contents as shown in Figure 4.

Within an individual slide, animations and audio are synchronized according to elapsed time, although as earlier explained, individual graphic components are only crudely animated in themselves. Provision is made to have long silences in the audio minimized to one second, and the annotations resynchronized, again using elapsed time. Further, within a slide, a mouse click will skip forward to the start of the next graphic animation, allowing rapid browsing of both the graphic elements and audio at the annotation level.

\section{5: Evaluation}

A pilot study of the use of the LLC software along with the LIDS system has been conducted. In this brief study the LLC software was used to capture the audio contents and annotations made during two lectures given to a class of around 20 students. The students were asked to compare the LIDS-based presentations with standard whiteboard-based presentations.

The majority of the participants found LIDS generally better than whiteboards. They were particularly positive about the fact that LIDS made it easy to change and annotate presentations to include, for instance, discussions initiated by the students.

The participants were also asked to compare LIDS with other presentations tools such as blackboards and overhead projectors. In this comparison LIDS was found to be at least as highly effective as the other presentation tools.

The students who took part in this study were encouraged to review the lecture notes online after their class presentations. Some of the student who reviewed the lecture presentations did so in order to review an entire lecture. Others just searched through the presentation to review particular areas they had not fully understood in the live presentations.

The length of time the students spent reviewing the material varied from ten minutes to four hours. Many participants felt that the ability to review the lecture presentations was beneficial. The most frequently identified benefit was the ability to access material missed in class. Other participants found the review ability useful in reinforcing or improving their understanding of the material covered. The flexibility to review material multiple times and at the participant's own pace were also mentioned. The audio was identified as a useful feature generally, and specifically in assisting with the understanding of the material reviewed.

\section{6: Conclusions}

This paper has described a prototype lightweight lecture capture system called LLC. LLC manages the showing of presentations prepared using Microsoft's PowerPoint $^{\mathrm{TM}}$ system. It provides mechanisms for capturing annotations made to slides during a presentation, as well as the audio content of the presentation. Most importantly LLC requires little or no effort additional to that normally made in preparing a lecture. Its only requirement is that the presenter use PowerPoint $^{\mathrm{TM}}$. In fact a range of presentation styles and levels of preparation is supported. A lecture can be delivered and recorded with no advance preparation at all. The presenter simply starts with a blank PowerPoint ${ }^{\mathrm{TM}}$ slide and writes or draws as they go, in the same way that they might with a conventional whiteboard. 
LCC adds all recorded information back into the presentation PowerPoint file, complete with timing data and animation settings to allow automatic replay of each slide, synchronising annotation with sound. As a result the LCC software in not required for replay. Students can use PowerPoint ${ }^{\mathrm{TM}}$ alone to review a lecture. A table of contents and timing components in the finished recording provide quick access to parts of the presentation when a student wants to review only part of the material. Review and redelivery can also be done using standard web browsers if the annotated lecture slides are converted to Web pages using LLC's HTML output utility.

A preliminary study of the LLC software in real lecture settings has demonstrated its effectiveness in capturing the annotations and audio contents of presentations. This study has also shown that the students have found LLC generated slides very useful for reviewing the contents of presentations after the lectures.

Further development of the LLC lecture audio and annotation capture and editing tools is currently underway. Experiments with speech recognition software are described in [2].

\section{7: Acknowledgements}

The research described in this paper has been carried out with the support of the Foundation for Research, Science and Technology under the New Economy Research Fund contract number UOWX9910.

\section{8: References}

1. Apperley, M, Dahlberg, B., Jeffries, A., Paine, L., Phillips, M., and Rogers, W. (2001), Applications of Large Interactive Display Surfaces, Proceedings of SIGCHI NZ Symposium on Computer Human Interaction, Palmerston North, New Zealand.

2. Apperley, M, Edwards, O, Jansen, S, Masoodian, M, McKoy, S, Rogers, B, Voyle, T, Ware, D. (2002); Application of Imperfect Speech Recognition to Navigation and Editing of Audio Documents, Proceedings of SIGCHI NZ Symposium on Computer Human Interaction, Hamilton, New Zealand.

3. Elrod, S., Bruce, R., Gold, R, Goldberg, D., Halasz, F., Janssen, W.; Lee, D., McCall, K., Pedersen, E., Pier, K., Tang, J., and Welch, B., (1992), Liveboard: A Large Interactive Display Supporting Group Meetings, Presentations and Remote Collaboration. Conference Proceedings of ACM SIGCHI 1992, 599-607.

4. Gehne, R., and Jesshope, C. (2000), Tools for the Production of Small-Footprint, Low-Bandwidth, Streaming Multi-Media for Distance Education, Conference Proceedings of Lifelong Learning 2000, 240244.

5. Jesshope, C. (2000), The Use of Multimedia in Internal and Extramural Teaching, Conference Proceedings of Lifelong Learning 2000, 257-262.

6. Smart Technologies (2002), http://www.smarttech.com/.

7. Virtual Ink Corporation (2002), http://www.mimio.com/. 